
Not for reproduction, distribution or commercial use.

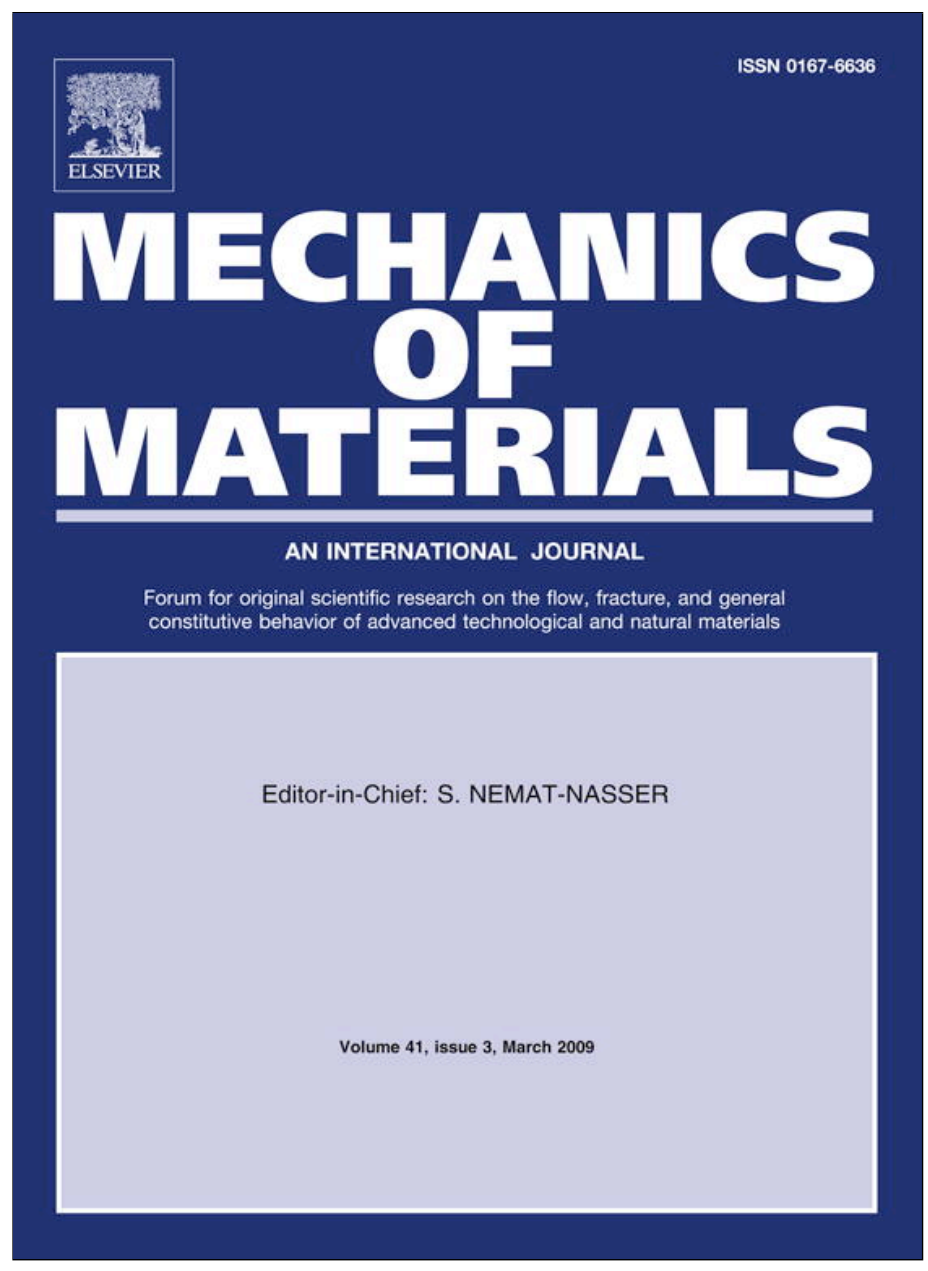

This article appeared in a journal published by Elsevier. The attached copy is furnished to the author for internal non-commercial research and education use, including for instruction at the authors institution and sharing with colleagues.

Other uses, including reproduction and distribution, or selling or licensing copies, or posting to personal, institutional or third party websites are prohibited.

In most cases authors are permitted to post their version of the article (e.g. in Word or Tex form) to their personal website or institutional repository. Authors requiring further information regarding Elsevier's archiving and manuscript policies are encouraged to visit:

http://www.elsevier.com/copyright 


\title{
A constitutive model for drying of a partially saturated porous material
}

\author{
Ivan Vlahinic ${ }^{a}$, Hamlin M. Jennings ${ }^{a, b, *}$, Jeffrey J. Thomas ${ }^{b}$ \\ ${ }^{a}$ Northwestern University, Department of Civil and Environmental Engineering, Evanston, IL 60208-3108, USA \\ ${ }^{\mathrm{b}}$ Northwestern University, Department of Materials Science and Engineering, Evanston, IL 60208-3109, USA
}

\section{A R T I C L E I N F O}

\section{Article history:}

Received 6 February 2008

Received in revised form 21 October 2008

\begin{abstract}
A B S T R A C T
A new constitutive model for drying of an elastic porous material is presented. The model is derived by decomposing the boundary value problem of drying into parts for which an explicit solution is readily available. The mathematical form differs from models developed using either the concept of equivalent pore pressure or average pore pressure. Instead of pressure averaging, the extent of weakening of the solid due to void inclusions, a material parameter, is found to be critical for the proper predictions of the volumetric strains. The model is applicable to drying under conditions where multi-layer adsorption of liquid persists, i.e. where the effects of solid surface energy are negligible, and where capillary pressure is the primary loading stimulus. A particular (and simple) form of the model is compared with experimental data for partially dried cement paste and Vycor $^{\mathrm{TM}}$ glass, and is shown to provide a much better agreement than previous models based on average pore pressure. Implications to the poromechanics and the effective stress of partially saturated porous media are discussed.
\end{abstract}

(c) 2008 Elsevier Ltd. All rights reserved.

\section{Introduction}

The constitutive behavior of porous materials has been studied extensively. In their pioneering works, Paul Fillunger in the 1910 s and Karl von Terzaghi in the 1920s explored the influence of pore fluid primarily on strength, but also on one-dimensional deformation of soils (see review by de Boer (1992)). In the late 1930s and 1940s, Biot (1941) developed a linear theory of poroelasticity. Since that time, both drained and undrained elastic behaviors (the two limit cases) of fluid-filled porous materials have been well grounded. The extension of Biot's theory to partially saturated porous materials has essentially remained unchallenged in the elastic region since Bishop (1959) introduced the concept of average pore pressure; and yet satisfactory agreement with experimental results has not been found even in relatively simple elastic materials such as Vycor ${ }^{\mathrm{TM}}$ glass (Bentz et al., 1998). And while

\footnotetext{
* Corresponding author. Address: Northwestern University, Department of Civil and Environmental Engineering, Evanston, IL 60208-3108, USA. Tel.: +1 8474915282; fax: +1 8474914011.

E-mail address: h-jennings@northwestern.edu (H.M. Jennings).
}

constitutive modelling of partially saturated materials continues to be a rich subject of ongoing research (e.g. Loret and Khalili, 2002; Gallipoli et al., 2003), with a comprehensive review on the subject given by Gens et al. (2007), the elastic formulation introduced by Bishop (1959) is seldom re-examined. Notable exceptions can be found in the contribution by Khalili et al. (2004) as well as in the models that are based on the assumption of multiple scales within the porous material (e.g. Chateau et al., 2002; Coussy and Brisard, in press).

The authors' modelling experience shows that experimental results of elastic shrinkage during drying of hardened cement paste are consistently and significantly greater than predictions by present formulations. Coussy et al. (2004) recently addressed the lack of agreement by modifying the capillary stress contribution to include an additional surface energy term. However, above 50\% relative humidity $(\mathrm{RH})$ capillary stresses should dominate surface energy effects due to multi-layer adsorption on the surfaces of emptied pores, and therefore it should be possible to obtain an accurate model using only the Kelvin-Laplace formulation for capillary stress. 
Here, a constitutive model is developed for partially saturated media subjected to drying, where pore pressure is known a priori. Vycor $^{\mathrm{TM}}$ glass and hardened portland cement paste (OPC) dried to various relative humidities are used as model materials. Cement paste in particular provides a unique model material because it has pores that span the nanometer to micron size range, allowing substantial variation in pore pressure due to capillary forces and fraction of pores filled. Thus, both the quantity of filled pores and pore pressure can be varied by drying to specific vapour partial pressures. Water saturation, the fractional quantity of water-filled pores, is determined by weight loss and the pressure is governed by the Kelvin-Laplace equation.

\section{General theory-effective stress and constitutive law}

\subsection{Fully saturated porous media}

The geomechanics community has developed the concept of 'effective stress' to model the behavior of soils. This facilitates computations by incorporating the influence of pore pressure, $p$, as well as external loads, $\sigma_{i j}$, into a single term, known as the 'effective stress', $\sigma_{i j}^{\prime}$. The problem of a fully saturated porous media is then treated like any other 'dry' continuum problem, such that:

$\sigma_{i j}^{\prime}=\sigma_{i j}-\zeta p \delta_{i j}$

Here, the positive values of the stress and pore pressure indicate compression. Parameter $\zeta$ reflects the fact that the pore pressure does not equally influence high and low porosity materials. Disagreement over its value permeated the literature well after Fillunger's and Terzaghi's introduction of effective stress and several different expressions for isotropic materials were proposed (see Table 1).

Terzaghi (1923) originally argued that $\zeta$ should be equal to the porosity, $\phi_{\circ}$, so that in the non-porous material the effect of pore pressure would vanish (Garg and Nur, 1973); and yet experimentally, he found $\zeta \simeq 1$ for several porous materials (Nur and Byerlee, 1971). Geertsma (1957), and later Skempton (1961), independently defined $\zeta$ in terms of $K_{\mathrm{b}}$, the (drained) bulk modulus of a porous material, and $K_{s}$, the bulk modulus of the solid or the grain. In this form, $\zeta$ is often referred to as the Biot coefficient. Suklje (1969) proposed another expression based on porosity, although rigorous proof was not provided. Nur and Byerlee (1971) later confirmed the result of Geertsma using a uniqueness theorem for the stress boundary value problem. For a general case of non-isotropic materials, the value of $\zeta$ depends on the constitutive material matrix (Zienkiewicz et al., 1999).

It is now instructive to write the constitutive equations for an isotropic fluid-saturated porous solid (Biot, 1962),

\section{Table 1}

Proposed $\zeta$ coefficients in 'effective stress' formulation Eq. (1).

\begin{tabular}{ll}
\hline$\zeta=\phi$ & Terzaghi (1923) \\
$\zeta=1-\left(K_{\mathrm{b}} / K_{\mathrm{s}}\right)$ & Geerstma (1957)/Biot (1962) \\
$\zeta=1-(1-\phi)\left(K_{\mathrm{b}} / K_{\mathrm{s}}\right)$ & Suklje (1969) \\
\hline
\end{tabular}

where $\mu_{\mathrm{b}}$ and $\lambda_{\mathrm{b}}$ are Lamé's constants of a dry material and $\varepsilon_{v}$ is a volumetric strain defined as $\varepsilon_{v}=\varepsilon_{x x}+\varepsilon_{y y}+\varepsilon_{z z}$ :

$\sigma_{x x}-\left(1-K_{\mathrm{b}} / K_{\mathrm{s}}\right) p=2 \mu_{\mathrm{b}} \varepsilon_{x x}+\lambda_{\mathrm{b}} \varepsilon_{v}$

$\sigma_{y y}-\left(1-K_{\mathrm{b}} / K_{\mathrm{s}}\right) p=2 \mu_{\mathrm{b}} \varepsilon_{y y}+\lambda_{\mathrm{b}} \varepsilon_{v}$

$\sigma_{z z}-\left(1-K_{\mathrm{b}} / K_{\mathrm{s}}\right) p=2 \mu_{\mathrm{b}} \varepsilon_{z z}+\lambda_{\mathrm{b}} \varepsilon_{v}$

$\sigma_{x y}=\mu_{\mathrm{b}} \varepsilon_{x y}$

$\sigma_{y z}=\mu_{\mathrm{b}} \varepsilon_{y z}$

$\sigma_{z x}=\mu_{\mathrm{b}} \varepsilon_{z x}$

From the constitutive equations, it is evident that pore pressure influences only normal strains (not shear), and hence only volumetric deformation (and not deviatoric). Physically, this conclusion also naturally stems from the fact that a fluid by definition cannot resist shear. ${ }^{1}$ Thus, absent of externally applied deviatoric stresses, the constitutive Eq. (2) can be reduced to Eq. (3), where a volumetric stress, $\bar{\sigma}$, is defined as $\bar{\sigma}=(1 / 3)\left(\sigma_{x x}+\sigma_{y y}+\sigma_{z z}\right)$ and where the bulk modulus can be expressed in terms of the Lamés constants of the dry material, i.e. $K_{\mathrm{b}}=(1 / 3)\left(2 \mu_{\mathrm{b}}+3 \lambda_{\mathrm{b}}\right)$ :

$\bar{\sigma}-\left(1-K_{\mathrm{b}} / K_{\mathrm{s}}\right) p=K_{b} \varepsilon_{v}$

It is often convenient to reorganize Eq. (3) and express the volumetric strain in terms of the applied stresses, as shown in Eq. (4). The latter constitutive equation appears to have been first published by Mackenzie (1950), although not in the context of the effective stress formulation.

$\varepsilon_{v}=\frac{\bar{\sigma}}{K_{\mathrm{b}}}-p\left(\frac{1}{K_{\mathrm{b}}}-\frac{1}{K_{\mathrm{s}}}\right)$

Thus far, only the drained and undrained responses of saturated materials have been examined. The saturated drained response corresponds to a state of zero excess pore pressure throughout the pore space. In this case pore fluid can flow in and out of the pore space without restriction and the material therefore deforms as if the pore fluid is not present. In the case of the saturated undrained response, fluid drainage is impeded and the excess pore pressure is finite.

\subsection{Partially saturated porous media}

In partially saturated porous materials, more than one fluid occupies the pore space and at least one fluid is under a finite pressure. The discussion here is limited to cases where air and water occupy all the porosity. For the majority of proposed formulations, the constitutive laws for the partially saturated system can be found directly from the fully saturated system. This is achieved by replacing the pore pressure term $p$ that appears in Eq. (4) with an effective pore pressure obtained by averaging the pressure of all the fluid constituents.

The first expression was developed by Bishop (1959), who averaged the pore pressures according to the contact area, $\chi$, between the fluid and the solid. Because of the inherent difficulty in quantifying the effective fluid contact area, $\chi$ is usually expressed as some function of the degree

\footnotetext{
1 This is true under quasi-static loading, i.e. where the shear stress components that result from viscous effects in a fluid are negligibly small.
} 
of fluid saturation, $S$, a quantity that can be conveniently measured, such that $\chi=\chi(S)$ for each fluid phase. The effective areas of water and air are related, i.e. $\chi_{w}+\chi_{a}=1$, and as a result the effective pore pressure can be described in terms of the effective water contact area only, $\chi_{w}$, commonly referred to as the Bishop parameter:

$p=\chi_{w} p_{w}+\left(1-\chi_{w}\right) p_{a}$

Instead of averaging the fluid contact areas, the formulation can be simplified by direct volume averaging. In his Ph.D. Thesis, this approach lead Schrefler to the same expression, with $\chi_{w}\left(S_{w}\right)=S_{w}$ where $S_{w}$ is the degree of water saturation, i.e. the volume fraction of pore space occupied by water; this work was later published by Lewis and Schrefler (1987). Hutter et al. (1999) came to a similar conclusion based on the thermodynamic theory of mixtures. This form has since been adopted by several investigators (e.g. Gallipoli et al., 2003; Coussy, 2004), and will be employed in this work for the purposes of model comparison. This is not to say that other expressions cannot be found in the literature, although they are typically derived on an empirical basis.

Absent of externally applied deviatoric stress, the constitutive law for a partially saturated system based on the effective pressure concept is shown below.

$\varepsilon_{v}=\frac{\bar{\sigma}}{K_{\mathrm{b}}}-\left(S_{w} p_{w}+\left(1-S_{w}\right) p_{a}\right)\left(\frac{1}{K_{\mathrm{b}}}-\frac{1}{K_{\mathrm{s}}}\right)$

\subsection{Generating pore pressures in a partially saturated system}

In a porous material, partial saturation can be introduced in several ways. Starting with a saturated specimen, load cells can be coupled with pumps to induce water egress and/or air entry and thus provide a great deal of control over the fluid phases (e.g. Sun et al., 2004). Instead of using pumps to generate suction pressures, water egress can also be induced via reverse osmosis. Furthermore, electrolysis (the ionization of hydrogen and oxygen when electric current is passed through the water) has been shown to allow for partial dewatering of the soils by introducing gas into the pore spaces (Yegian et al., 2007).

In contrast to the above mentioned experimental methods, drying provides perhaps the simplest way of generating partial saturation in a porous material. As pore water is removed by lowering the external relative humidity, capillaries form at the fluid-fluid interface and control the value of the pore pressure. This means that the pore pressure need not be experimentally measured as, at equilibrium, it can be solved a priori via the Kelvin-Laplace equation shown in Eq. (7), with radius of meniscus, $r_{m}$, defined in Eq. (8) and variables described in Table 2.

$p_{c}=-(R T / M) \ln (h)$

$r_{m}=2 \gamma / p_{c}$

In the case of the materials examined in this work, the two fluids are water and air, while the corresponding capillary pressure, $p_{c}$, is defined as the difference between air pressure and water pressure, i.e. $p_{c}=p_{a}-p_{w}$. Furthermore,
Table 2

Description and constants used in Kelvin-Laplace Eq. (7).

\begin{tabular}{lll}
\hline Constant & Symbol & Value unit \\
\hline Surface tension & $\gamma$ & $0.0728 \mathrm{~N} / \mathrm{m}$ \\
Molar volume & $M$ & $1.802 \times 10^{-5} \mathrm{~m}^{3} / \mathrm{mol}$ \\
Univ. gas constant & $R$ & $8.314 \mathrm{Nm} / \mathrm{Kmol}$ \\
Abs. temperature & $T$ & $293 \mathrm{~K}(\mathrm{OPC})$ \\
Relative humidity & & $284 \mathrm{~K}($ glass $)$ \\
& $h$ & $0-100 \%$ \\
\hline
\end{tabular}

$\left|p_{a}\right| \ll\left|p_{w}\right|$ as the absolute value of the pressure in the water quickly exceeds atmospheric pressure during drying, climbing above $10 \mathrm{MPa}$ (100 atm) at only 92\% RH. Considering now the case of no external load, the constitutive law Eq. (6) reduces to:

$\varepsilon_{v}=S_{w} p_{c}\left(\frac{1}{K_{\mathrm{b}}}-\frac{1}{K_{\mathrm{s}}}\right)$

Bentz et al. (1998) applied Eq. (9) to Vycor ${ }^{\mathrm{TM}}$ glass and found that it significantly underestimates the measured deformations below $80 \%$ relative humidity.

\section{New constitutive formulation-drying of a partially saturated system}

\subsection{Constitutive law for a fully saturated porous system}

Following the method of Nur and Byerlee (1971), we first derive the constitutive law for a fully saturated porous system. The porous system is composed of a linear elastic solid with connected pores of arbitrary shape. The pores are assumed homogeneously distributed.

For simplicity, we consider the porous system subject to a uniform pore pressure $p=p_{c}$ and free of any external loads. This boundary value problem is largely intractable on its own, but becomes tractable when decomposed into individual parts for which an explicit solution is readily available. Hence we consider a convenient superposition of stresses, as shown in Fig. 1a. We first apply an external hydrostatic compression $\bar{\sigma}=p_{c}$, which results in a strain of $p_{c} / K_{b}$ (note that compression is defined positive). Next, we apply an external hydrostatic pressure $\bar{\sigma}=-p_{c}$ and an equal pore pressure $p=-p_{c}$. This results a uniform stress in the solid (e.g. Love, 1944, p.86) and a strain equal to $-p_{c} / K_{s}$.

Since we assume the porous system exhibits linear elastic behavior, the strain fields resulting from the two steps can be added and the constitutive law reduced to:

$\varepsilon_{v}=p_{c}\left(\frac{1}{K_{\mathrm{b}}}-\frac{1}{K_{\mathrm{s}}}\right)$

Invoking a uniqueness theorem for the stress boundary value problem, Nur and Byerlee (1971) used the preceding superposition of stresses to deduce a theoretically exact expression for the effective stress. Note that Eq. (10) is identical to Eq. (4), absent of externally applied loads.

\subsection{Constitutive law for a partially saturated porous system}

We now apply a similar strategy to a partially saturated porous system, where only select pores are subject to a 
a



b

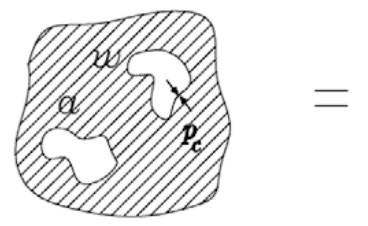

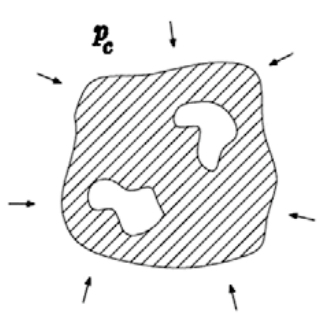

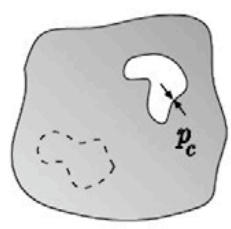

$p_{c} \quad+$

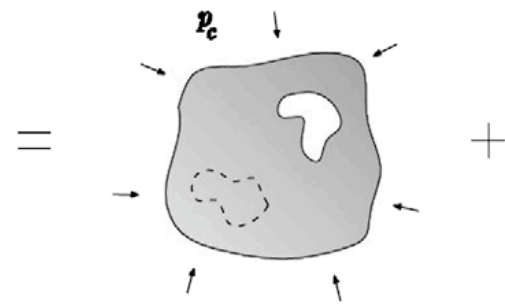

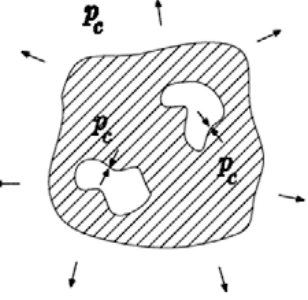

homogeneous elastic solid

'effective' hom. elastic solid

porous region occupied by air ' $a$ ' or water ' $w$ '

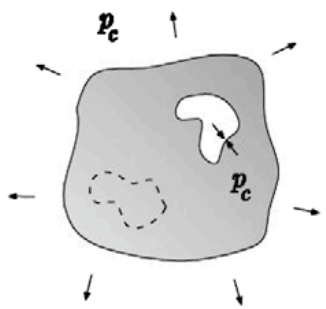

Fig. 1. Stress superposition schematic of (a) fully saturated and (b) partially saturated system. Capillary pressure, $p_{c}$, is shown to act on the surface of the internal solid boundary. Influence of atmospheric air pressure is assumed inconsequential.

uniform pore pressure $p=-p_{c}$. Conceptually, we define a boundary of a new 'effective solid' delineated by the surfaces of pores that apply pressure, i.e. the remaining full pores. In this manner, we can reduce a partially saturated system to a fully saturated system, provided we update the material constants of the new solid. Physically, the load imposed by the capillaries is still carried by the solid, although the solid is now weakened and behaves as if it were porous itself.

To arrive at the solution, we similarly decompose the boundary value problem described above into two loading steps as shown in Fig. 1b. In the first step, we apply positive (normal) tractions $\bar{\sigma}=p_{c}$ on the external boundary of the porous body. It is apparent that under this loading, the volumetric strain is equivalent to that caused by external load $p_{c}$ in a fully drained porous system and is equal to $p_{c} / K_{b}$ regardless of the degree of drying. In the second step, we impose equal tractions on internal and external boundaries. This results a uniform state of stress in the effective (weakened) solid and strain of $-p_{c} / \bar{K}$. The 'effective bulk modulus', $\bar{K}$ describes the bulk modulus of the effective solid and varies during drying. It can be conveniently expressed in terms of the degree of water saturation, $S_{w}$, as the volume of water-filled and empty pores are closely related. Finally, combining the strain fields, the constitutive law of a partially saturated system reduces to:

$\varepsilon_{v}=p_{c}\left(\frac{1}{K_{\mathrm{b}}}-\frac{1}{\bar{K}\left(S_{w}\right)}\right)$

Before proceeding, a brief check of the behavior at extremes should be performed. In the case of zero saturation, water occupies negligible porosity. Hence, $\bar{K} \rightarrow K_{\mathrm{b}}$ as evident from Fig. 1b, and the volumetric strain given by Eq. (11) reduces to zero as expected. In the case of full saturation, water occupies all the pores, $\bar{K} \rightarrow K_{\mathrm{s}}$, and Eq. (11) correctly reduces to Eq. (10).

Similar linear superposition of stresses can be established for systems that include external loads as well as two or more fluid phases where each fluid is under finite pressure. Presently, for simplicity, we include only a 2phase fluid system free of external boundary stresses and assume the influence of the gas phase (air) to be inconsequential.

From Eq. (11), it is clear that the constitutive law for a partially saturated system does not necessitate the use of pressure averaging over all fluid constituents, in direct contrast to Eq. (9). Rather, the extent of weakening of the effective solid skeleton, a material parameter, must be deduced to determine the volumetric deformation. This is consistent with the experimental observation that the Bishop parameter $\chi$ is often found to be strongly influenced by the material fabric (Coleman, 1962).

It should also be noted that in the geomechanics community, it is customary to assume that the solid or grain is incompressible. In a fully saturated system, this assumption has a clear meaning, namely that $K_{\mathrm{b}} \ll K_{\mathrm{s}}$ when $S_{w}=1$. As such, the assumption also allows for a natural simplification of Eq. (10). In a partially saturated system, however, the assumption of solid incompressibility does not easily simplify Eq. (11) because, in general, the effective bulk modulus $\bar{K}$ cannot be assumed incompressible even if $K_{\mathrm{b}} \ll K_{\mathrm{s}}$. This is because even a few pores can give 
rise to an appreciable compressibility in an otherwise incompressible medium.

\subsection{Quantifying the loss of stiffness of the effective solid}

Before analyzing the influence of void inclusions on bulk properties of the effective solid, it is useful to relate how the porosity of the effective solid varies as a function of the degree of saturation. To this end, we define a volume fraction, $n^{\alpha}$, of the solid, water, and air phases, with $\alpha=s, w, a$ correspondingly. We further note that the degree of (water) saturation can be expressed as $S_{w}=n^{w} /\left(n^{w}+n^{a}\right)$ and that the fraction of the material occupied by the air and water phases, the total porosity, is given by $\phi_{\circ}=n^{w}+n^{a}$. At any stage of drying, the porosity of the effective solid, $\varphi$, then amounts to $\varphi=n^{a} /\left(n^{s}+n^{a}\right)$ (see Fig. 1b) and in terms of the degree of saturation simplifies to:

$\varphi\left(S_{w}\right)=\frac{\left(1-S_{w}\right) \phi_{\circ}}{1-S_{w} \phi_{\circ}} \quad$ where $\quad 0 \leqslant \varphi\left(S_{w}\right) \leqslant \phi_{\circ}$

Now only the influence of porosity on the solid compressibility remains to be quantified. Before proceeding, it should be mentioned that what is referred to as porosity in the effective solid is in fact the space that is occupied by the gas phase (air). While it may be argued that air may offer a role of structure and thus limit the compressibility of the effective solid, this role is largely negligible; the compressibility of a solid is typically orders of magnitude less than that of gas. Therefore, for the purpose of estimating the effective solid properties, the gas (air) phase may be treated as a void or a cavity rather than a material phase with finite compressibility.

The experimentally derived relationships can certainly provide the solid properties for varying values of porosity. Oftentimes, however, such relationships are not readily available and theoretical approaches may need to be employed. The simple rule of mixtures can establish upper and lower bounds for parallel (Voight) and series (Reuss) configurations. Unfortunately, as void inclusions are infinitely compressible, the lower bound estimate invariably yields $\bar{K}=0$ for any value of porosity, a conclusion that also applies to the classic Hashin-Strickman lower bound (Hashin, 1983).

Using a concept of 'averaged stress', many successful micromechanical schemes have been built on the foundations of the Eshelby (1957) tensor. Among these, perhaps the most prominent are the Mori-Tanaka (e.g. Mori and Tanaka, 1973; Weng, 1984) and self-consistent (e.g. Hershey, 1954) approximations. The former have proven useful in a variety of composite materials while the latter have found success with the porous materials whose skeleton is polycrystalline or granular; for example, Constantinides and Ulm (2007) recently found the self-consistent scheme useful in modeling the elastic behavior of cement-based materials at the lower material scales. For a more detailed treatment of the aforementioned schemes (and others), Christensen (1990) provides a good overview and comparison.

Predictions can be greatly improved when values of $K_{\mathrm{b}}$ and $K_{\mathrm{s}}$ are established experimentally and used as

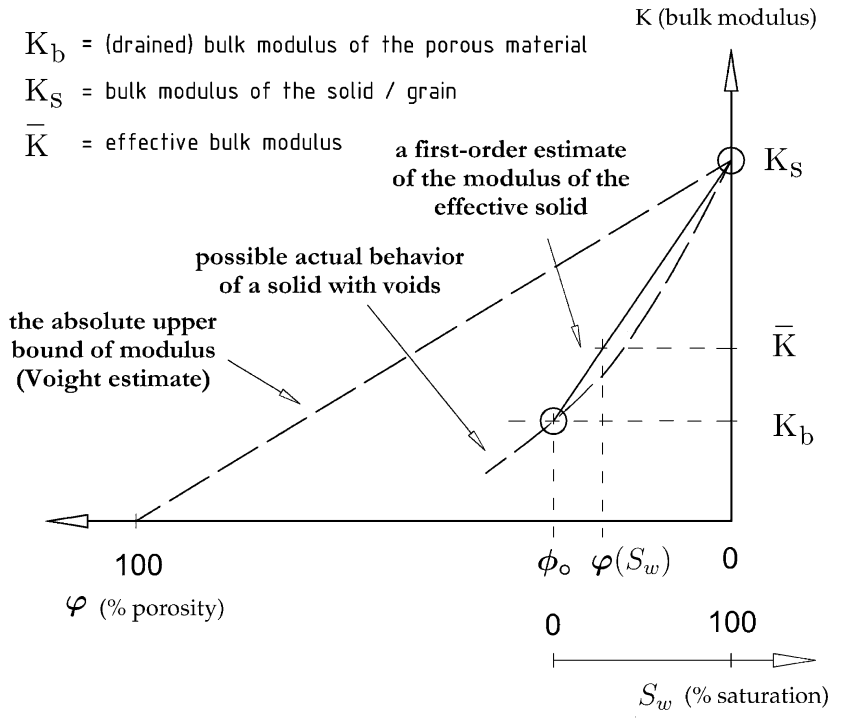

Fig. 2. Effect of void inclusions on elastic properties of a solid. In absence of an actual relationship, a first-order estimate of the properties of the "effective solid" can be established using the experimentally measured values of the drained bulk modulus $K_{\mathrm{b}}$ and the solid bulk modulus $K_{\mathrm{s}}$.

bounds thereafter. From Fig. 2, it is evident that a straight line between the two experimentally measured moduli yields a first-order estimate of the effective properties for the porous material in question. In this regard, low porosity materials enjoy an inherent advantage: as porosity decreases, the first-order estimate approaches the true properties of the effective medium. The experimentally aided estimate of $\bar{K}$ is given by:

$\bar{K} \approx K_{\mathrm{s}}-\frac{K_{\mathrm{s}}-K_{\mathrm{b}}}{\phi_{\circ}} \varphi\left(S_{w}\right)$

It should be noted that Eq. (13) may not provide a good estimate for solids whose effective properties are affected greatly when voids are introduced. One such limitation may be especially prominent in solids often referred to as "incompressible" for which $K_{\mathrm{b}} \ll K_{\mathrm{s}}$ as noted earlier. For these materials, voids clearly have a detrimental effect on the overall volumetric stiffness, as evidenced by the very fact that $K_{\mathrm{b}}$ is orders of magnitude smaller than $K_{\mathrm{s}}$. A large loss of stiffness can be captured by micromechanical estimates if highly irregular pore shapes, e.g. crack-like cavities, or a high concentration of voids are present. In such cases, a better estimate than the one shown in Eq. (13) should be used for more accurate predictions of volumetric deformations via Eq. (11); however, this is beyond the scope of this work.

\section{Experimental comparison}

\subsection{Drying strains of hardened cement paste on second drying}

Our formulation can be perhaps most easily compared against the experimental results of a partially dried material, which provides favorable conditions for several reasons. The induced capillary stresses are purely hydrostatic. For a 
homogeneous and isotropic materials the deformations then translate to uniform strains in all directions, avoiding a necessity of volumetric measurements. Linear deformation, i.e. drying shrinkage, in any one direction equates to $1 / 3$ of the total volumetric deformation. Differential shrinkage, which gives rise to cracking, can be avoided by slow step-wise drying, i.e. by gradually lowering the relative humidity to the desired final value, and by the use of specimens with small cross section.

Because a new equilibrium can be obtained for each partial saturation, only these values of length change at each partial vapour pressure need to be recorded experimentally. At equilibrium, exchange of pore liquid with the environment also stops. By recording weight difference between successive relative humidity points, preferably for the entire range of saturation states, the degree of saturation $S_{w}$ in the porous system may be interpolated.

It is very important to note that hardened cement paste does not deform elastically the first time it is dried as the first drying cycle certainly induces permanent dimensional changes. However, each successive cycle is nearly fully reversible in terms of water sorption and shrinkage, as shown experimentally by e.g. Helmuth and Turk (1967), Verbeck et al. (1968), and others. It is on this basis that elastic shrinkage formulations may be compared against experimental data using the 2 nd drying cycle of pastes.

Published data on 2nd drying of cement paste is scarce. Recent experiments at Northwestern are starting to fill this void. For example, data on pastes with water to cement ratio by mass of 0.5 has been acquired by Gevrenov (2005). The water retention curve ${ }^{2}$ is displayed in Fig. 3; each data point is an aggregate of measurements from several specimens. ${ }^{3}$

\subsubsection{Material properties of hardened cement paste}

Before comparing shrinkage models with experiments, a brief review of available data on elastic constants is necessary, as their values are not readily available. Direct measurements of the bulk modulus of the solid constituent $K_{\mathrm{s}}$ of cement paste with varying $\mathrm{w} / \mathrm{c}$ ratios and ages were performed by Grasley et al. (2006). A small scatter of data was observed, with values of $K_{\mathrm{s}}$ in the range of 40-50 GPa. Fortunately, such variation does not result in significant changes in the model predictions of shrinkage strain (up to $5 \%$ ). Therefore, $K_{\mathrm{s}}=45 \mathrm{GPa}$ is used here.

Values of $K_{\mathrm{b}}$, on the other hand, significantly influence the final shrinkage values and, therefore must be used judiciously. Grasley et al. (2006) calculate $K_{\mathrm{b}}$ within the range of $8.3-9.0 \mathrm{GPa}$, using an approach presented in its companion publication (Scherer, 2006). Estimates of $K_{\mathrm{b}}$ can also be made based on available tests of Young's

\footnotetext{
2 The water retention curve is shown as a function of relative humidity. From the displayed data, the values of the capillary pressure may be deduced via Eq. (7).

3 Data on the 1st drying cycle is available for comparison in literature. In particular, careful measurements by Roper (1966) compare favorably to those of Gevrenov (2005), where both 1st and 2nd drying cycles are obtained using the same experimental methods. This provides additional confidence in the referenced data set.
}

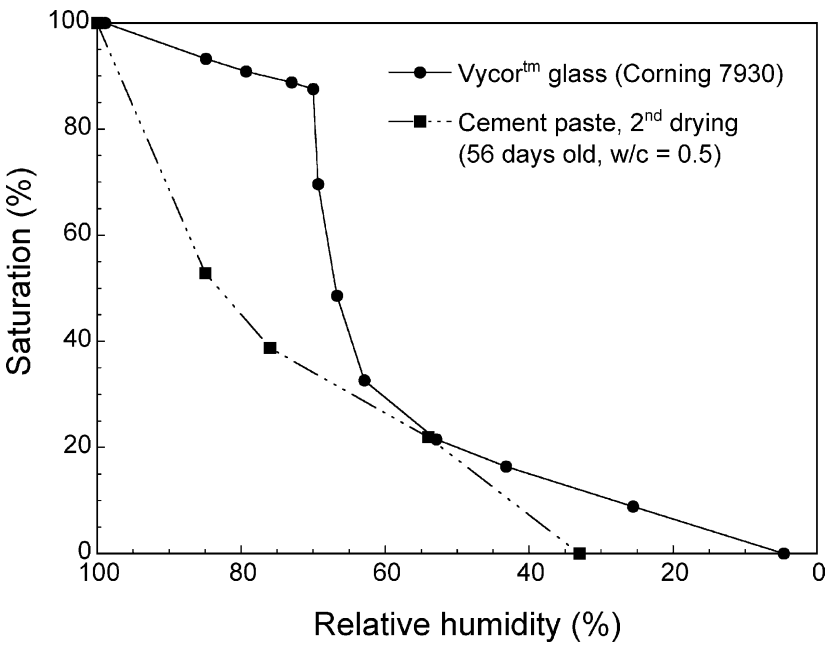

Fig. 3. Water retention data for $\operatorname{Vycor}^{\mathrm{TM}}$ glass (Corning 7930) and hardened cement paste (2nd drying, 56 days old, $w / c=0.5$ ) based on experiments by Amberg and McIntosh (1952), Gevrenov (2005) respectively. In Vycor $^{\mathrm{TM}}$ glass water retention is fully reversible for all drying cycles while in hardened cement paste it is nearly fully reversible after the first drying cycle. Percent saturation was interpolated from the weight the samples lost on drying to specific relative humidities. See text for more details.

modulus and Poisson's ratio. Measurements by Ghosh and Timusk (1974), which are consistent with those of Feldman (1968), show $E_{\mathrm{b}} \simeq 12$ GPa for Young's modulus of hardened paste with $w / c=0.5$. Poisson's ratio, as reported by Parrott (1974) for $w / c=0.5$, was shown to vary between $v_{\mathrm{b}}=0.220-0.275$, depending on the degree of saturation. These numbers correspond to $K_{\mathrm{b}}=6.9-8.6 \mathrm{GPa}$, slightly lower than those estimated by Grasley et al. (2006). Because of the comparatively large effect of bulk modulus on final shrinkage predictions, the two extremes of $K_{\mathrm{b}}=7 \mathrm{and} K_{\mathrm{b}}=9 \mathrm{GPa}$ are included in our predictions.

How bulk modulus varies with porosity remains uncertain. At present, the only known studies of the influence of porosity on paste modulus were performed by Feldman (1968) who found that hardened cement paste exhibits a semi-logarithmic relationship between elastic modulus and porosity. Unfortunately, the published data set contains two significant shortcomings:

(1) The author fails to indicate the method with which porosity of paste was measured. This issue is of critical importance due to sensitivity of results to experimental methods and controversies over methods of measuring capillary porosity (Jennings, 2008). Sensitivity lies in the dominant constituent of hardened cement paste, calcium silicate hydrate $(\mathrm{C}-\mathrm{S}-\mathrm{H})$ which is highly hydrophylic and contains structural water.

(2) Modulus data in the porosity range below 20\% was not measured directly, a range most useful at present. Instead, it was interpolated from higher porosity cement paste samples.

For these reasons, applicability of Feldman's conclusions to this work remains uncertain. Instead, an interpola- 
tion of effective bulk modulus based on the experimental values of $K_{\mathrm{b}}$ and $K_{\mathrm{s}}$ will be used. For detailed discussion of the method, refer to Section 3.3.

\subsubsection{Types of water in cement paste and applicability to capillary tension}

Water plays a critical role in hardened cement paste, and its presence (or lack thereof) defines essentially all of its material properties. For this reason, it is important to address the influence of different water types.

Due to the chemical properties and hydrophilic nature of $\mathrm{C}-\mathrm{S}-\mathrm{H}$, not all water remaining in hardened cement paste during drying is under hydrostatic stress. Chemically combined water in $\mathrm{C}-\mathrm{S}-\mathrm{H}$ is, in fact, part of the solid structure. ${ }^{4}$ It is non-evaporable, and certainly not subject to capillary pressures. Water that is adsorbed onto highly hydrophilic layers and surfaces of $\mathrm{C}-\mathrm{S}-\mathrm{H}$ also does not maintain all the properties of bulk water due to solid surface forces.

Beyond the reach of surface forces, water in pores behaves as bulk and thus maintains the full capillary pressure $p_{c}$ imposed by the meniscus. It is established experimentally that on average, one molecular layer of water coats the surfaces of hardened cement paste at $11 \% \mathrm{RH}$, and two layers at 35\% RH (Badmann et al., 1981; Jennings, 2008). Adsorption of additional layers results in negligible change in solid surface energy (e.g. Mindess and Young, 1981, p.490). This suggests that, during drying, an estimate of water subjected to capillary stress can be obtained by measuring the water that evaporates from the sample between full saturation and 35\% relative humidity. The degree of water saturation can then be estimated by dividing the current weight change of the sample from saturation $(100 \% \mathrm{RH})$ by the weight change on drying to $35 \%$ RH.

In this work, we define $33 \% \mathrm{RH}$ as a zero saturation point. This value is used simply for convenience because it is part of the referenced data set. In addition, we find the total (capillary) porosity to be $\phi_{\circ}=0.28$ (Tennis and Jennings, 2000).

\subsection{Drying strains of Vycor $^{\mathrm{TM}}$ glass}

In contrast to hardened cement paste, $\mathrm{Vycor}^{\mathrm{TM}}$ glass (Corning 7930) has an extremely narrow distribution of pore sizes, with an overwhelming majority of the pores on the order of nanometers and with the pores of micron size nearly entirely absent ( Nordberg, 1944; Scherer, 1986). This provides a distinct model material in which the separation of scale between the different porous regions is largely absent.

Due to its morphology, the porous glass tends to retain the saturating liquid during the initial stages of drying while precipitously losing much of the liquid in a narrow range of relative humidity values, as evident by the water retention data displayed in Fig. 3. Desorption and adsorption isotherms also start to merge near the lower end of this range, indicating a loss of capillaries. The detailed dry-

\footnotetext{
${ }^{4}$ The water content of solid $\mathrm{C}-\mathrm{S}-\mathrm{H}$ was recently determined by Allen et al. (2007).
}

ing experiments by Amberg and McIntosh (1952) place the loss of capillary stability near $60 \%$ relative humidity.

The desorption isotherms of $\mathrm{Vycor}^{\mathrm{TM}}$ glass are also fully reversible for all drying cycles, as shown by Nordberg (1944), Amberg and McIntosh (1952), and others, suggesting that all measured strains are fully elastic. The dynamic Young's and shear moduli were measured by Scherer (1986) who found that $E_{\mathrm{b}}=14.7 \mathrm{GPa}$ and $G_{\mathrm{b}}=6.34 \mathrm{GPa}$ respectively; this corresponds to $K_{\mathrm{b}}=7.2 \mathrm{GPa}$. In the literature, no indication exists that anyone has ever directly measured the properties of the glass skeleton. The work by Bentz et al. (1998) suggest values of $E_{\mathrm{s}}=45 \mathrm{GPa}$ and $v_{\mathrm{s}}=0.1$ for the Young's modulus and Poisson's ratio of the solid respectively based on micromechanical analysis, while Amberg and McIntosh (1952) suggest values of $E_{s}=40 \mathrm{GPa}$ and $v_{\mathrm{s}}=0.25$. Presently, we use $v_{\mathrm{s}}=0.20$, a value that is typical of most glass types, along with an average of the reported values of Young's moduli; this yields $K_{\mathrm{s}} \approx 23.6 \mathrm{GPa}$. The manufacturer reports a total porosity value of $\phi_{\circ}=0.28$.

The composition of Vycor ${ }^{\mathrm{TM}}$ glass is fundamentally different than that of cement paste, in that water is not an integral part of the solid phase. Here we define a zero saturation point at $5 \%$ relative humidity, where on average slightly more than one molecular layer coats the surface of the glass (Emmett, 1942); this RH point is also a convenient part of the referenced data set. As very little water is lost from Vycor ${ }^{\mathrm{TM}}$ glass at such low humidity values, the influence of the zero saturation value on the predicted values of strain is small.

\subsection{Predictions of the proposed drying model}

Combining the proposed constitutive law given in Eq. (11) with the expressions for $p_{c}$ and $\bar{K}$ defined in Eqs. (7), (12) and (13), the drying shrinkage in any one direction reduces to Eq. (14). Similarly, the drying shrinkage based on the constitutive law using an averaged pore pressure Eq. (9) together with Eq. (7) reduces to the classic Biot-Bishop constitutive law Eq. (15):

$$
\begin{aligned}
\varepsilon_{s h} & =-\frac{1}{3} \frac{R T}{M} \ln (h)\left(\frac{1}{K_{\mathrm{b}}}-\frac{1}{\bar{K}}\right) \\
& \text { where } \bar{K} \approx K_{\mathrm{s}}-\frac{\left(K_{\mathrm{s}}-K_{\mathrm{b}}\right)\left(1-S_{w}\right)}{\left(1-S_{w} \phi_{\mathrm{o}}\right)} \\
\varepsilon_{s h} & =-\frac{1}{3} \frac{R T}{M} \ln (h) S_{w}\left(\frac{1}{K_{\mathrm{b}}}-\frac{1}{K_{\mathrm{s}}}\right)
\end{aligned}
$$

In Figs. 4 and 5, we compare the predictions ${ }^{5}$ of our new formulation Eq. (14), with that of the classic averaged pore pressure model Eq. (15). The proposed model exhibits much better agreement with experimental data for two very distinct materials. That the pore structures of the materials greatly differ from each other is evident by the fraction of water each material retains at a specific relative humidity point, as shown by the water retention data in Fig. 3. The

\footnotetext{
5 The predictions are based on the first-order estimate of the effective solid properties, aided by the experimentally measured bounds (see Section 3.3). This estimate may need to be improved if porosity is found to significantly influence the overall volumetric stiffness of the effective solid.
} 


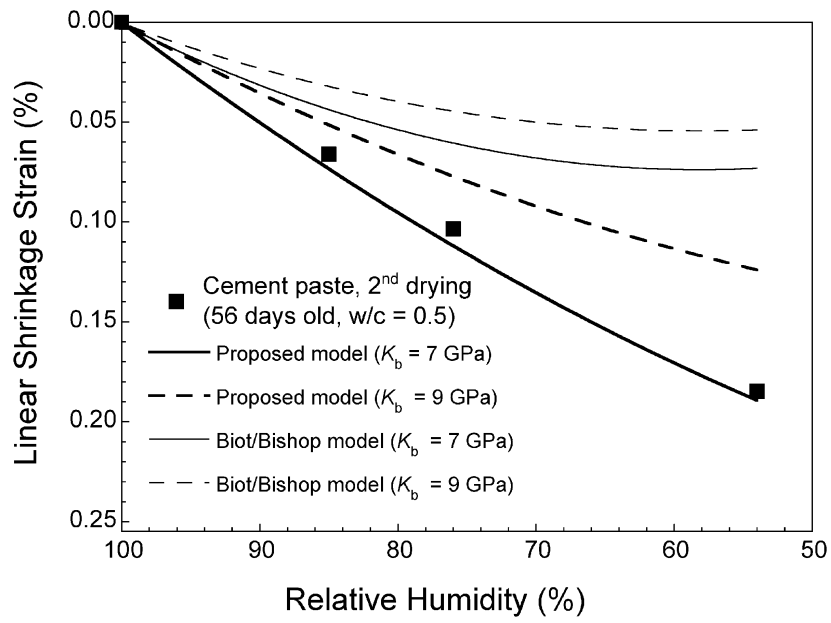

Fig. 4. Experimental data for 56-day-old paste Gevrenov, 2005 with predicted drying shrinkage curves. Each model is shown for $K=7 \mathrm{GPa}$ and $K=9 \mathrm{GPa}$, i.e. limits of reported bulk modulus values; $K_{\mathrm{s}}=45 \mathrm{GPa}$. Zeropoint bulk water saturation is assumed at $33 \%$.

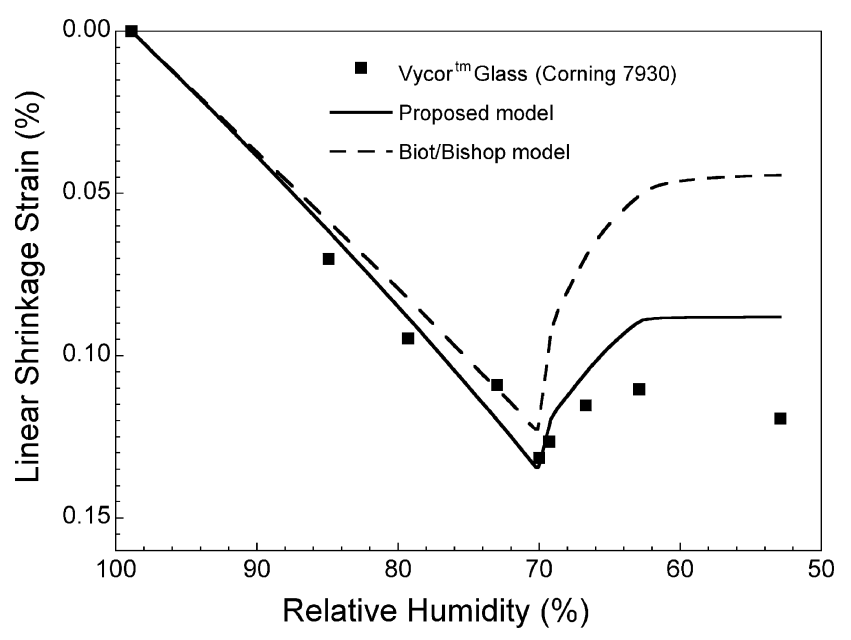

Fig. 5. Experimental data for $\mathrm{Vycor}^{\mathrm{TM}}$ glass (Amberg and McIntosh, 1952) with predicted drying shrinkage curves. Each model is shown for $K=7.2 \mathrm{GPa}$ and $K_{\mathrm{s}}=19 \mathrm{GPa}$, modulus of the bulk and the solid skeleton, respectively. Zero-point bulk water saturation is assumed at 5\%; an exact value is of little influence on predicted strain values in glass.

predicted strain values for $K_{\mathrm{b}}=7$ and $K_{\mathrm{b}}=9 \mathrm{GPa}$ fall on either side of the data in cement paste and the predicted strain values for Vycor $^{\mathrm{TM}}$ glass are able to better capture the trend of the measured data.

Figs. 4 and 5 also show the extent to which the classic model Eq. (15) underpredicts experimental measurements. The disagreement is especially prominent when the materials are truly partially saturated. This approximately corresponds to relative humidity range below $75 \%$ in both materials, precisely the range in which capillary mechanisms should be the most dominant. While the proposed model still appears to underpredict the large drying strains captured by the experiments, albeit somewhat less so in the case of hardened cement paste, the improvement over the classic model is readily apparent.

Recently, a new formulation was proposed by Coussy et al. (2004) that accounts for the effects of surface energy in cementitious materials. It was suggested that surface energy serves to augment capillary effects, resulting in deformations up to $30 \%$ greater than those predicted by the classic Biot-Bishop model at 50\% relative humidity. However in light of the discussion in Section 4.1.2, the contribution of the solid surface energy to deformation is likely negligible above $50 \%$ relative humidity due to the presence of adsorbed water.

It should further be added that it has long been recognized that the complete mechanical behavior of partially saturated materials cannot be described adequately via use of the effective stress alone (a tensorial variable) and that an additional variable (a scalar) is often required. In the case of the elastic material behavior, the need for an additional scalar variable largely stems from the observations that capillary pressure appears to augment the force of contact between the adjacent particles or grains (e.g. Bishop and Eldin, 1950). As the (normal) contact force between grains increases due to capillary pressure, so does the ability of the grains in contact to withstand shear forces (and sometimes higher tensile stresses). It is noted that while for some materials this effect is extremely important (e.g. one can build castles out of partly wet sand whereas a highly saturated sand and even dry sand crumbles under its own weight) for others it is less so (e.g. relative humidity appears not to affect the shear strength of cement paste, although it does affect fracture properties (Beaudoin, 1982).

In this work, we present a new constitutive model for the volumetric response of a porous material during drying. From the macroscopic arguments, we suggest a modification to the classic form of the constitutive law, and by extension to the classic form of the effective stress, generally a tensorial variable. The increase in shear resistance due to capillaries is inherently a micromechanical phenomenon and is not captured by the present formulation just as it is not captured by the classic Biot-Bishop expression herein described. In the simplest way, the increased shear resistance can often be accounted for via an additional (scalar) variable which depends on the capillary pressure (suction) as introduced by e.g. Gallipoli et al. (2003). In the case of inelastic material behavior, additional considerations are likely needed. The basic arguments present in this work intend to illuminate the subject in the context of linear elasticity, which forms a theoretical basis for the more advanced constitutive models.

\section{Conclusion}

By decomposing a boundary value drying problem, a new constitutive model is developed to predict the elastic shrinkage due to capillary stresses during drying of a porous material. The model is valid under conditions where solid surface energy does not play an important role in deformations and where capillary stresses due to airwater menisci are present. For materials discussed in this work, this translates to relative humidity values above about $50 \%$ and $60 \%$ for hardened cement paste and $\mathrm{Vycor}^{\mathrm{TM}}$ glass, respectively. 
The predictions of the new model were evaluated against experimental measurements of linear drying strains. Hardened cement paste and $\mathrm{Vycor}^{\mathrm{TM}}$ glass were chosen as model materials for their distinct pore size distributions and relatively large shrinkage strains that ease collection of experimental data. Additionally, cement paste exhibits nearly elastic behavior during the 2nd drying cycle and $\mathrm{Vycor}^{\mathrm{TM}}$ glass during all drying cycles. Good agreement was found above $50 \%$ relative humidity for cement paste, while the predictions were much improved above $60 \%$ for porous glass, without use of any other energy terms, whereas the classic model based on average pore pressure significantly underpredicts the measured drying strains for both materials.

The form of effective stress in partially saturated porous media is also discussed in light of the present formulation. It is found that a constitutive relation for a porous system infiltrated by more than one fluid does not necessitate pore pressure averaging, as is the case for all previous formulations. Instead, a material parameter needs to be evaluated, the extent of weakening of the "effective solid" that results from partial emptying of pores (this is in agreement with experimental observations that the Bishop parameter $\chi$ is often found to be strongly influenced by the material fabric). In turn, it is required that the influence of voids on the solid properties are known. Presently, we estimate these properties with the help of experimentally measured limit values. Improved estimates of the effective solid properties may be needed for materials containing a solid phase whose compressibility is significantly affected by the presence of the voids.

\section{Acknowledgements}

This work was supported by the National Science Foundation under Grant CMS 0409571. The authors would also like to thank Zdenek Bažant, Franz-Josef Ulm, Jose Andrade, and Dale Bentz for their critical thoughts during preparation of the manuscript.

\section{References}

Allen, A., Thomas, J., Jennings, H., 2007. Composition and density of nanoscale calcium-silicate-hydrate in cement. Nature Materials 6 (4), 311-316.

Amberg, C., McIntosh, R., 1952. A study of adsorption hysterisis by means of length changes of a rod of porous glass. Canadian Journal of Chemistry 30 (12), 1012-1032.

Badmann, R., Stockhausen, N., Setzera, M.J., 1981. The statistical thickness and the chemical potential of adsorbed water films. Journal of Colloid and Interface Science 82 (2), 534-542.

Beaudoin, J.J., 1982. Effect of humidity and porosity on fracture of hardened portland cement. Cement and Concrete Research 12 (6), 705-716.

Bentz, D.P., Garboczi, E.J., Quenard, D., 1998. Modelling drying shrinkage in reconstructed porous materials: Application to porous vycor glass. Modelling and Simulation in Material Science and Engineering 6, 211-236.

Biot, M.A., 1941. General theory of three-dimensional consolidation. Journal of Applied Physics 12, 155-164.

Biot, M.A., 1962. Mechanics of deformation and acoustic propagation in porous media. Journal of Applied Physics 33 (4), 1482-1498.

Bishop, A., 1959. The principle of effective stress. Teknisk Ukeblad 39 $859-863$.

Bishop, A., Eldin, A., 1950. Undrained triaxial tests on saturated sands and their significance in the general theory of shear strength. Geotechnique 2 (1), 13-32.
Chateau, X., Moucheront, P., Pitois, O., 2002. Micromechanics of unsaturated granular media. Journal of Engineering Mechanics of ASCE 128 (8), 856-863.

Christensen, R., 1990. A critical evaluation of a class of micro-mechanics models. Journal of the Mechanics and Physics of Solids 38 (3), 379404.

Coleman, J.D., 1962. Stress-strain relations for partially saturated soils. Geotechnique 12 (4), 348-350.

Constantinides, G., Ulm, F.-J., 2007. The nanogranular nature of C-S-H. Journal of Mechanics and Physics of Solids 55, 64-90.

Coussy, O., 2004. Poromechanics, second ed. Wiley-VCH, Berlin.

Coussy, O., Brisard, S., in press. Prediction of drying shrinkage beyond the pore iso-deformation assumption. Journal of Mechanics of Materials and Structures.

Coussy, O., Dangla, P., Lassabatère, T., Baroghel-Bouny, V., 2004. The equivalent pore pressure and the swelling and shrinkage of cementbased materials. Materials and Structures 37 (1), 15-20.

de Boer, R., 1992. Development of porous media theories-a brief historical review. Transport in Porous Media 9, 155-164.

Emmett, P., 1942. Measurement of Surface Areas of Finely Divided or Porous Solids by Low-Temperature Adsorption Isotherms in Advances in Colloid Science, vol. 1. Interscience Publishers Inc., New York.

Eshelby, J.D., 1957. The determination of the elastic field of an ellipsoidal inclusion and related problems. Proceedings of the Royal Society of London Series A 241 (6), 376-396.

Feldman, R., 1968. Structure and physical properties of cement pastes. In: Proceeding of the 5th International Symposium on the Chemistry of Cement, vol. 3. Tokyo, Japan, p. 53.

Gallipoli, D., Gens, A., Sharma, R., Vaunat, J., 2003. An elasto-plastic model for unsaturated soil incorporating the effect of suction and degree of saturation on mechanical behaviour. Geotechnique 53 (1), 123-135.

Garg, S., Nur, A., 1973. Effective stress laws for fluid-saturated porous rocks. Journal of Geophysical Research 78, 5911-5921.

Geertsma, J., 1957. The effect of fluid pressure decline on volumetric changes of porous rocks. Transactions of AIME 210, 331.

Gens, A., Sánchez, M., Sheng, D., 2007. On constitutive modelling of unsaturated soils. Acta Geotechnica 1 (3), 137-147.

Gevrenov, J. S., 2005. Microstructure and drying shrinkage of ordinary portland cement and alkali-activated slag pastes as a function of relative humidity Master's Thesis, Northwestern University.

Ghosh, R.S., Timusk, J., 1974. Effect of sustained loading at early ages on the modulus of elasticity of cement paste. Materials and Structures 7 (5), 335-340.

Grasley, Z.C., Scherer, G.W., Lange, D.A., Valenza, J.J., 2006. Dynamic pressurization method for measuring permeability and modulus: II Cementitious materials. Materials and Structures 40 (7), 711-721.

Hashin, Z., 1983. Analysis of composite materials-a survey. Journal of Applied Mechanics 50.

Helmuth, R., Turk, D., 1967. The reversible and irreversible drying shrinkage of hardened portland cement and tricalcium silicate pastes. Journal of the Portland Cement Association, Research and Development Lab 9 (2), 8-21.

Hershey, A., 1954. The elasticity of an isotropic aggregate of anisotropic cubic crystals. Journal of Applied Mechanics 21, 236-240.

Hutter, K., Laloui, L., Vulliet, L., 1999. Thermodynamically based mixture models of saturated and unsaturated soils. Mechanics of CohesiveFrictional Materials 4, 295-338.

Jennings, H.M., 2008. Refinements to colloid model of C-S-H in cement: CM-II. Cement and Concrete Research 38, 275-289.

Khalili, N., Geiser, F., Blight, G.E., 2004. Effective stress in unsaturated soils: review with new evidence. International Journal of Geomechanics 4 (2), 115-126.

Lewis, R., Schrefler, B., 1987. The Finite Element Method in The Deformation and Consolidation of Porous Media. John Wiley and Sons, Chichester.

Loret, B., Khalili, N., 2002. An effective stress elastic-plastic model for unsaturated porous media. Mechanics of Materials 34, 97-116.

Love, A., 1944. A Treatise on The Mathematical Theory of Elasticity, 4th ed. Dover, New York.

Mackenzie, J.K., 1950. Elastic constants of a solid containing spherical holes. Proceedings of the Physical Society Section B 63 (1), 2-11.

Mindess, S., Young, F.J., 1981. Concrete. Prentice-Hall, Englewood Cliffs, NJ.

Mori, T., Tanaka, K., 1973. Average stress in matrix and average elastic energy of materials with misfitting inclusions. Acta Metallurgica 21, 571-574.

Nordberg, M., 1944. Properties of some vycor-brand glasses. Journal of the American Ceramics Society 27, 299-305. 
Nur, A, Byerlee, J, 1971. An exact effective stress law for elastic deformation of rock with fluids. Journal of Geophysical Research 76, 6414-6419.

Parrott, L.J., 1974. Lateral strains in hardened cement paste under short- and long-term loading. Magazine of Concrete Research 26 (89), 198-202.

Roper, H., 1966. Dimensional change and water sorption studies of cement paste. Symposium on Structure of Portland Cement Paste and Concrete. Washington, D.C., pp. 74-83.

Scherer, G.W., 1986. Dilatation of porous glass. Journal of the American Ceramics Society 69 (6), 473-480.

Scherer, G.W., 2006. Dynamic pressurization method for measuring permeability and modulus: I theory. Materials and Structures 39 (10), 1041-1057, 1059.

Skempton, A., 1961. Effective stress in soils, concrete, and rock. In: Proceedings of Conference on Pore Pressure and Suction in Soils. Butterworth, London, pp. 4-16.

Suklje, L., 1969. Rheological Aspects of Soil Mechanics. Wiley, New York. p. 123.

Sun, D.A., Matsuoka, H., Xu, Y.F., 2004. Collapse behavior of compacted clays by suction controlled triaxial tests. ASTM Geotechnical Testing Journal 27 (4), 362-370.
Tennis, P.D., Jennings, H.M., 2000. A model for two types of calcium silicate hydrate in the microstructure of portland cement pastes. Cement and Concrete Research 30 (6), 855-863.

Terzaghi, K.v., 1923. Die berechnung der durchlassigkeitsziffer des tones aus dem verlauf der hydrodynamischen spannungserscheinungen. Sitzungsberichte der Akademie der Wissenschaften Wien $132,125$.

Verbeck, G. J., Helmuth, R. H., 1968. Structure and physical properties of cement pastes. In: Proceeding of the 5th Inetrnationa Symposium on the Chemistry of Cement, vol. 3. Tokyo, Japan, pp. $1-32$

Weng, G.J., 1984. Some elastic properties of reinforced solids, with special reference to isotropic ones containing spherical inclusions. International Journal of Engineering Science 22 (7) 845-856.

Yegian, M.K., Eseller-Bayat, E., Alshawabkeh, A., Ali, S., 2007. Inducedpartial saturation for liquefaction mitigation: Experimental investigation. Journal of Geotechnical and Geoenvironmental Engineering 133 (4), 372-380.

Zienkiewicz, O., Chan, A., Pastor, M., Schrefler, B., Shiomi, T., 1999. Computational Geomechanics. John Wiley and Sons, Chichester. 